\title{
Design of An Intelligent Hierarchical System for Fingerprint Management of Electricity Loads Based on Operating Characteristics of Household Appliances
}

\author{
Zhukui Tan ${ }^{1}$, Lefeng Cheng ${ }^{2 *}$, Chao Ding ${ }^{1}$, Weifeng Xu², Zhengjia Li ${ }^{2}$, Lijin Zhao $^{1}$, Guangyu Xu ${ }^{2}, \mathrm{Xufeng} \mathrm{Yuan}^{3}, \mathrm{Bin}_{\mathrm{Liu}}{ }^{1}$, \\ Wenjing Huang ${ }^{2}$ \\ ${ }^{1}$ Electric Power Research Institute of Guizhou Power Grid Co., Ltd, Guizhou, Guiyang 550002, China \\ ${ }^{2}$ Suzhou Huatian Power Technology Co., Ltd, Jiangsu, Suzhou 215000, China \\ ${ }^{3}$ Guizhou University, Guizhou, Guiyang 550025, China
}

\begin{abstract}
In order to facilitate the active identification capability of various types of electrical equipment, so as to enhance the functions such as the behavioural analysis and optimization control of electricity utilization in an automatic demand side management (DSM) system. A definition is given on load fingerprint based on operating characteristics of household appliances, as well as its architecture. In addition, based on the cyber-physical systems (CPS) technology, a hierarchical technical framework for load fingerprint management is proposed. This framework is a two-layer electricity utilization load fingerprint management platform, including local management and cloud-based management. Finally, two hardware prototypes are developed, including a socket-type smart collection terminal and an intelligent interactive concentrator with multiple communication technologies, which can provide strong hardware support for the realization of the hierarchical technical framework proposed in this paper.
\end{abstract}

\section{Introduction}

With the rapid development of modern power systems, new concepts such as smart grid, energy Internet, energy router, automatic demand response, and smart load dispatching have been proposed by scholars in recent years. Taking smart grid as an example, each consumer node and even specific electricity utilization equipment can be monitored and controlled in the smart grid, which can realize the interconnection and intercommunication of information and energy between all nodes in the entire power grid [1-2]. The development of smart grid has promoted the innovation of traditional electricity consumption modes, and flexible and interactive smart electricity utilization systems have gradually taken shape [3]. Based on the electricity price signals issued by the electricity market (EM) and combined with their own power consumption information, the power users can actively respond to the economic incentives on the distribution network side, so as to regulate their conventional power consumption patterns and achieve the optimal allocation of user power consumption under the demand side response mechanisms [4-5].

In order to realize the functions of real-time and automation of demand-side management (DSM), key techniques need to be developed to support this requirement. In other words, it is essential to support an intelligent monitoring system that has been penetrated into every end-user and every electricity device and is able to support real-time measurement, two-way communication, and feature recognition. Among them, the feature recognition refers to that the system is able to actively identify the types and identity features of electrical equipment, which is conducive to autonomous and intelligent management for the monitoring system.

In recent years, investigations on smart electricity utilization system have been focused on design of individual smart communication terminals [6-8], or advanced measurement systems [9], which is lack of research work about active identification of electricity loads based on electrical characteristics of electrical equipment. Among current investigations, Y.C. Ke [10] investigated the load characteristic and response potential of several typical household appliances, but he did not apply it to the device's feature recognition. Q.Q. Liu [11] designed a non-intrusive residential electricity utilization visualization system based on load characteristics. However, this system does not have a database of load characteristics of various types of equipment and it cannot be employed to effectively identify the newly accessed equipment type. To address it, Y.X. Yu et al. [12] proposed a non-intrusive household electricity load identification method, but it can only be used to separate a given set of equipment, which cannot meet such a household electricity utilization environment with complicated and varied household appliances.

\footnotetext{
* Corresponding author: Lefeng Cheng, chenglf_scut@163.com
} 
Cyber-physical system (CPS) is a new generation of intelligent system that embeds information-acquisition technology, IntelliSense technology, and network communication technology on the basis of traditional physical systems to realize the flow and sharing of information within the system [4, 13-14]. In this paper, based on the CPS technologies [15-18], we give a concise definition on load fingerprint, that is, we define the characteristics of the load embodied by a powered device in a specific operating mode as its load fingerprint.

Based on the concept of load fingerprint, in order to solve the issue that the engineering applications of nonintrusive detection technology are only limited to the established equipment, and further facilitate active identification of different types of equipment under the DSM system, we proposed a CPS-based hierarchical system architecture design scheme for load fingerprint management. On this basis, we established a design concept for a two-layer fingerprint management platform involving local management and cloud-based management, and further developed key hardware equipment to support this architecture. In this system scheme, the fingerprint data collected by the developed hardware equipment can be employed to identity the load characteristics to support analysis of electricity consumption behaviour for users and power utilization optimization and control under automatic demand response (ADR).

\section{Load fingerprint and fingerprint grading}

\subsection{Load fingerprint definition}

From start-up to stable operation, electrical equipment contains load characteristics that meet certain laws during this process, which is determined by its internal circuit structure and work rules [19]. As defined earlier, the load fingerprint of a kind of equipment refers to the load characteristics reflected by this electricity utilization equipment in a specific operating mode. Taking the active power as an example, as shown in Figure 1, in which the variation of active power of a household load in an entire process from the start to the stable operation is illustrated. In this figure, the operating area after this equipment is started can be divided into a transient section and a steady-state section. Here, the transient section is a transition zone of load power, that is, the power oscillation process from the start of the equipment to stable operation.

For different types of equipment, there is a large difference in the power waveform of their transient sections, thus we define the load characteristic embodied in this section as transient load fingerprint. For the steady-state section, the active power remains basically unchanged in this section, or changes in certain rules, such as air-conditioners, refrigerators, and other intermittent operating electrical appliances, thus we refer to the load characteristic embodied in the steady-state operating section as the steady-state load fingerprint of the load.

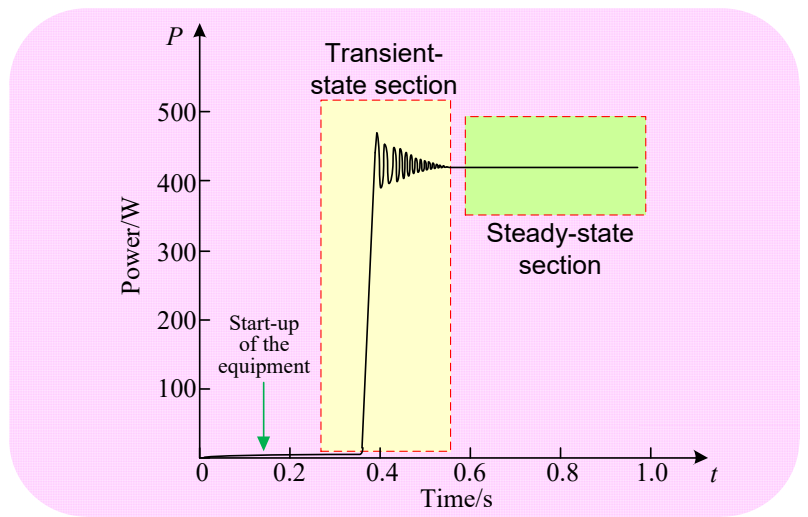

Fig. 1. The variation curve of active power of a household load in an entire process from the start to the stable operation.

Generally speaking, the steady-state and transientstate load fingerprints constitute a two-stage load fingerprint, which is regarded as a set of load fingerprints for the load. The load fingerprint of a type of load is not fixed, which is related to a specific working mode, for example, the air-conditioner has a variety of working conditions such as cooling and heating, and the electric fans have different gears. For this type of electrical appliances with multiple states, the number of load fingerprint sets is the same as the number of working states. In other words, the number of load fingerprint sets for the $N$-state appliances is $N$.

\subsection{Fingerprint grading}

As stated previously, the load fingerprint contains transient-sate fingerprint and steady-state fingerprint. On this basis, the fingerprint grading of them is briefly introduced as follows.

\subsubsection{Steady-state fingerprint grading}

When the electricity equipment operates normally in a specific mode, its steady-state current of this equipment has a certain statistical rule under the condition that the power supply voltage meets the requirements of the national standard. For resistive electrical appliances and inductive electrical appliances, their current curves are generally similar to sine waves. While for electronic appliances, the current curve of them appears as a periodic non-sinusoidal wave. Therefore, we can define the steady-state load fingerprint of the electrical equipment from the perspective of steady-state current curve. We can use a type of power sampling equipment with a sufficiently high sampling frequency to sample the steady-state current and voltage of a load in a normal working state. We assume that the sampling values of the voltage and current at each point are $V_{n}$ and $I_{n}$, respectively, the sampling frequency is $f$, the number of continuous sampling cycles is $m$, and the number of sampling points in one cycle is $N$. Thereby, the calculation methods of the voltage RMS value and current RMS value are as follows: 


$$
\begin{gathered}
V_{\mathrm{rms}}=\sqrt{\sum_{n=1}^{N} \frac{V_{\mathrm{n}}^{2} \times 50}{m f}} \\
I_{\mathrm{rms}}=\sqrt{\sum_{n=1}^{N} \frac{I_{\mathrm{n}}^{2} \times 50}{m f}}
\end{gathered}
$$

According to (1) and (2), we can further calculate some important parameters that can be employed to characterize the steady-state characteristics of the load, such as active power, reactive power, power factor, and apparent power. Hence, we can incorporate the voltage RMS value, current RMS value, active power, reactive power and power factor into the steady-state load fingerprint set corresponding to the operating mode. Since the apparent power can be calculated from the above parameters, it does not need to be included.

Generally, there are significant differences in the current harmonic characteristics of different types of electricity utilization equipment. For example, the harmonic components of electronic appliances are extremely abundant; the odd harmonics of motor-driven electrical appliances are more obvious; while the currents of pure resistive electrical appliances such as heaters do not contain harmonic components. The steady-state operating current of the load is the superposition of the fundamental wave component, the DC component, and a series of harmonic components. Addressed concretely, the Fast Fourier Transformation (FFT) can be employed to decompose the current detection signals in the same operating mode, and the number of harmonics is calculated to 31 times. In addition, the DC component, the amplitude of the current fundamental wave, and 2 31 times current harmonic amplitudes are also included in the set of load fingerprints.

\subsubsection{Transient-state fingerprint grading}

For different types of household appliances, the transition time required for the operation from onset to steady state is different. There are also variations in the electrical parameters such as current and power during this period of time. For example, TVs and desktops have a long open process, usually between 1 2 seconds, and they have significant current pulses; air-conditioners and washing machines have obvious current and power oscillations after they are turned on; heaters and other similar electrical appliances have a short switching process, and their steady states can be reached within about $0.3 \mathrm{~s}$ without significant current pulse and power oscillation. Therefore, we can define the transient load fingerprint based on the transient characteristics when the power appliances are turned on. Firstly, we should select the peak current pulse with obvious characteristics and the transient duration time as the basic indexes of transient load fingerprint.

For TVs, there will be two short peaks at two different moments in one second after they are turned on. For the refrigerators, when they are turned on, there will be a peak process lasting about $1 \mathrm{~s}$. Therefore, taking into account the very different power waveforms of different loads, it is essential to apply the load fingerprint in the engineering application of household load identification. However, a satisfactory recognition rate cannot be achieved only by the aforementioned steady-state load fingerprint and two transient feature parameters. We need to use the power quality analysers to conduct a lot of measurement statistics, in order to determine that the instantaneous load characteristic of action time of most electrical appliances is within $2 \mathrm{~s}$ when they are turned on.

In addition, we can use the intelligent acquisition terminal to start the sampling according to the load step threshold. Concretely, we can intercept a transient signal with a duration of $2 \mathrm{~s}$ to calculate the active power waveform vector as $P(n)=\left[p_{1}, p_{2}, \ldots \ldots p_{n}\right]$ and reactive power waveform vector as $Q(n)=\left[q_{1}, q_{2}, \ldots \ldots q_{n}\right]$, where $p_{1} \sim p_{n}$ and $q_{1} \sim q_{n}$ are the instantaneous active and reactive power of each sampling point, respectively. We determine the active waveform vector and reactive waveform vector as two important indexes of transient load fingerprint.

\section{Hierarchical technical framework design}

\subsection{Hardware system framework}

As stated previously, CPS is a new-type interconnected system integrating large-scale computing systems, sensor networks, communication networks, and control systems. It has the functions of real-time monitoring, analysis, and control of interconnected physical systems. Therefore, in order to realize the collection and management of load fingerprints under distribution network conditions, we start from the perspective of CPS, try to embed the necessary intelligent hardware devices in the traditional household distribution system and have designed a load fingerprint management technology framework, as graphically in Figure 2.

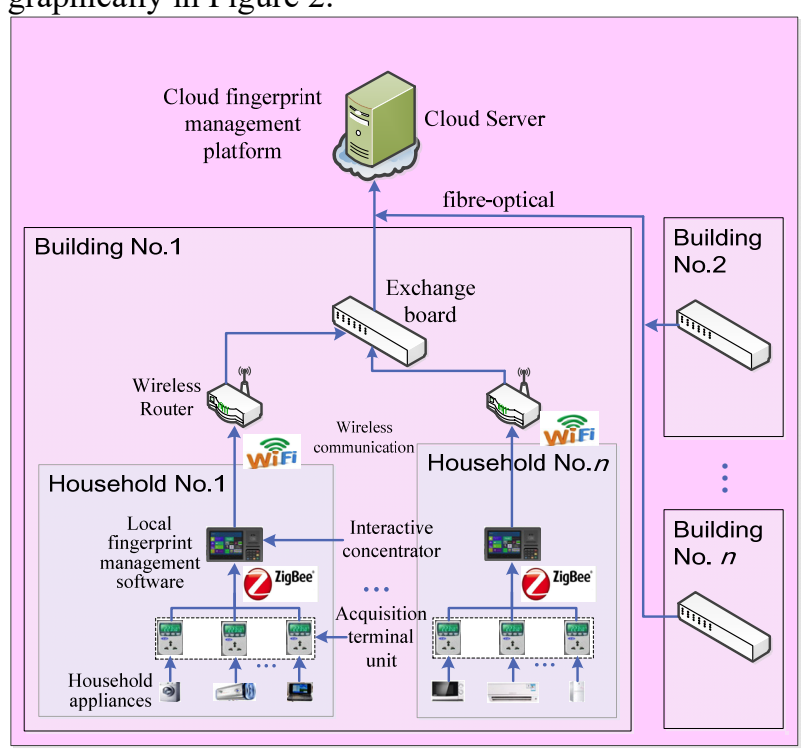

Fig. 2. Framework design of the hardware system.

In Figure 2, for each electrical device, the smart socket-type data acquisition terminal unit is installed and connected to the distribution network. Each collection 
terminal is used to obtain the load fingerprint data of the electricity utilization devices and then through the ZigBee wireless communication network, the terminal can exchange data with the unique smart interactive concentrator in the electricity user who owns this terminal. Here, the smart interactive concentrator is responsible for identifying and applying the load fingerprints of all the power devices in this household user, so as to realize the collection and localized management of the load fingerprints. The intelligent interactive concentrator of each user finally can be used to upload the load fingerprint data to the cloud fingerprint management platform and application platform via a WIFI manner to complete the second layer of load fingerprint management and application.

\subsection{Function of each layer}

According to the framework design of the hardware system presented in Figure 2, this load fingerprint management hardware system framework can be divided into four functional layers from the bottom to the top, including electrical equipment layer, data-aware and acquisition layer, centralized control and interaction layer, and cloud platform layer. The adjacent layers can perform data communication and coordinate with each other to achieve the collecting, analyzing, storing, and identifying of the load fingerprint data. The above four layers are briefly introduced as follows.

\subsubsection{Electrical equipment layer}

This layer is a generic term for various typical household appliances and can be regarded as the data source layer for providing various types of equipment load fingerprints. When the electrical appliances are connected to the distribution network through the collection terminal for power transmission, the collection terminal also acquires and transmits the data such as the voltage, current, power, harmonics, transient duration, and other load fingerprint information of the electricity appliances, thereby realizing the internal input and circulation of the fingerprint data in the system.

\subsubsection{Data-aware and acquisition layer}

The hardware device in this layer is mainly a socket type smart collection terminal. These terminals have functions such as data acquisition, data analysis, wireless communication, and control response. The acquisition terminal has two functions: one is the energy interface between the distribution network and the electrical appliances, and the other is the acquisition of fingerprint data. The acquisition terminal can support micro-power wireless communication technology, namely ZigBee technology, to complete the transfer of fingerprint data to a local interactive concentrator and real-time response concentrator control instructions.

In order to ensure accurate acquisition of load transient information, the data-aware acquisition layer has the following requirements: the sampling frequency of the device must not be less than $6.4 \mathrm{kHz}$ in general; the $\mathrm{ADC}$ resolution must be at least 12 bits; at the same time, in order to make the control commands respond in a timely manner, ZigBee technology wireless communication module must meet the following working conditions: the data transmission rate should be guaranteed to be above $250 \mathrm{kbit} / \mathrm{s}$, the response delay is not higher than $30 \mathrm{~ms}$, and the error rate of continuously sending multiple data packets is controlled within $1 / 1000$.

\subsubsection{Centralized control and interaction layer}

This layer of hardware equipment is mainly an intelligent interactive concentrator. The concentrator can support a variety of wireless communication technologies such as ZigBee and $\mathrm{Wi}-\mathrm{Fi}$, and is responsible for identifying and managing load fingerprints of all appliances belonging to the user. The concentrator controls the acquisition terminal through ZigBee communication and receives the load fingerprint data transmitted by the latter. In addition, the local fingerprint management software built into the concentrator can perform preliminary processing and identification of the fingerprint data, and then analyze results and two transient fingerprints, including active power waveform vector and the reactive power waveform vector with large amount of data will be uploaded to the cloud server through WiFi communication.

\subsubsection{Cloud platform layer}

This layer is the top layer within the load fingerprint management architecture. We build a load fingerprint management platform on the cloud server to store waveform vector data of various devices in a slice area, and finally implement integrated management of load fingerprints, analysis of power usage patterns, and optimization control of power usage.

\subsection{Framework design of two-layer fingerprint management platform}

As shown in Figure 3, we build load fingerprint management and application software platforms at the interactive concentrator layer and the cloud platform layer, respectively.

The Android software of the intelligent interaction concentrator monitors the input of the fingerprint data in real time through the data interface. This ensures that on the one hand the steady-state load fingerprint and the current pulse peak and transient duration in the transient load fingerprint are stored in its SQLite database to build a local fingerprint database. At the same time, data mining can be performed by using load theory, decision tree, machine learning, and other relevant theoretical methods to achieve fingerprint recognition, so as to obtain the type of electrical appliances running in the household and their electricity consumption conditions, and to use the identification results for electricity utilization behaviour analysis based on electricity 
utilization patterns, and optimization and control of electricity utilization based on multi-objective optimization method. On the other hand, the intelligent interactive concentrator uploads the local analysis result, the active power waveform vector, and the reactive power waveform vector to the cloud server via the Internet.

As shown in Figure 3, the cloud server receives the feature data and analysis results uploaded by the intelligent interactive concentrator and stores it in the background MySQL database. After the load fingerprint management and application platform established in the cloud acquires data from the fingerprint database, the data is applied to the following four functional modules:

- Data monitoring and electricity analysis module: based on the electrical data monitoring, the load characteristics are analysed, and the electricity consumption patterns of each user are constructed to formulate power consumption optimization strategies.

- Power quality and energy efficiency analysis module: including harmonic analysis, voltage deviation analysis, etc., and based on the energy efficiency index system after screening and optimization, comprehensive energy efficiency assessment for power users can be achieved.

- Optimization and control module: According to the results of the load fingerprint recognition, the power demand of various types of power load, such as rigid demand type load and elastic demand type load, used by the power user can be obtained. Moreover, the intelligent power optimization control can be performed in combination with the result of the power consumption pattern analysis.

- Message service module: the module can manage the power users and their power consumption information in an integrated manner, and publish demand side announcements based on incentive information such as electricity prices and policies.

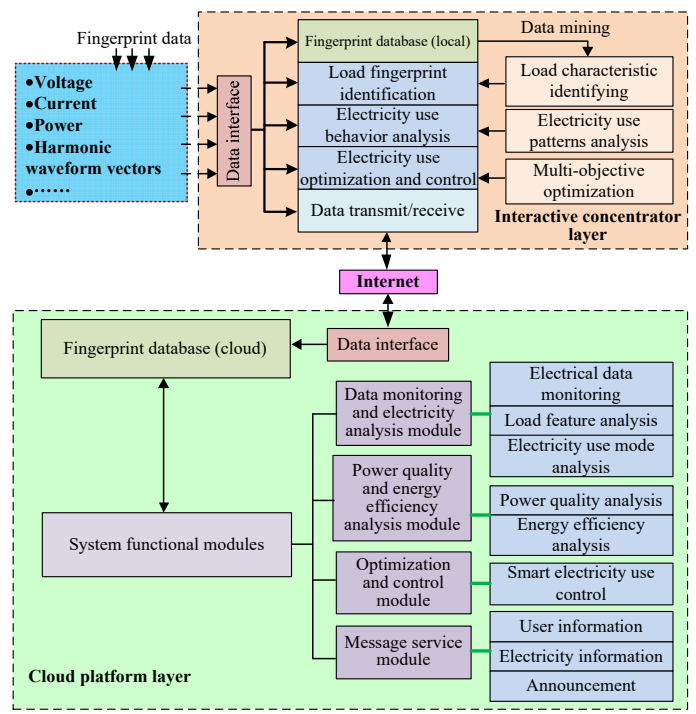

Fig. 3. Framework design of two-layer fingerprint management platform.

\section{Hardware development}

\subsection{Socket-type intelligent collection terminal development}

In order to implement the CPS-based load fingerprint management architecture described above, the actual engineering application first needs to develop a sockettype smart collection terminal that is suitable for common household appliances as a collector of the load fingerprint data and a responder of upper-level control commands. Taking into account that the acquisition terminal needs to perform high-frequency sampling and processing such as FFT harmonic analysis and other complex operations, this paper uses a digital signal processing chip (DSP) as a central processing unit to design a socket-type smart acquisition terminal hardware structure, as shown in Figure 4.

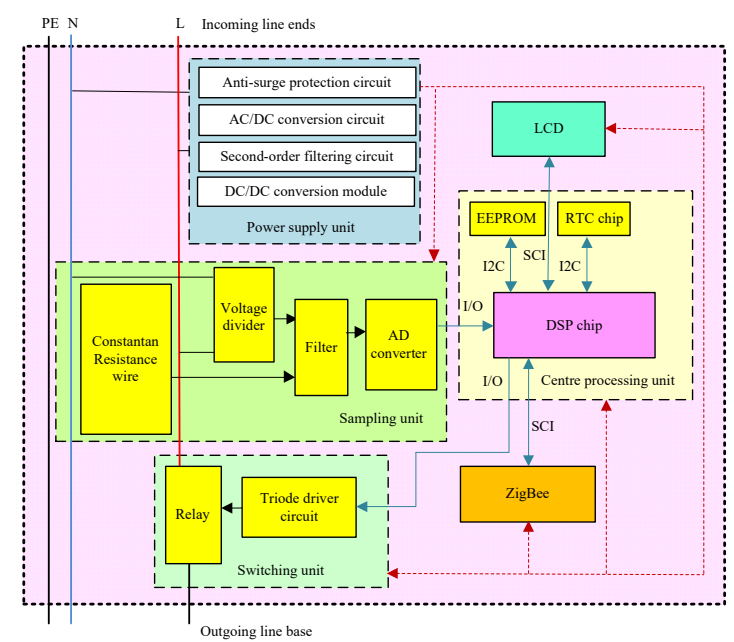

Fig. 4. Hardware structure design of the intelligent collection terminal.

The intelligent acquisition terminal uses a DSP chip as the core processor, and is responsible for data processing, communication interaction, and coordination of the functions of stable operation of each module. The central processing unit is equipped with an off-chip EEPROM and RTC chip through an $\mathrm{I}^{2} \mathrm{C}$ interface, which is used to store key parameters and add time flag bits to the test data.

The constantan resistance wire is connected in series to the single-phase $220 \mathrm{~V}$ zero line to convert the load current into a small voltage signal. A precision resistor divider circuit is used in parallel with the circuit to obtain the voltage sampling value. The two sampling signals are filtered through an RC low-pass filter circuit to remove the high frequency. After the component, it is input to the DSP chip through the A/D conversion circuit for load fingerprint calculation. This DSP chip is the CPU of the intelligent acquisition terminal, which is mainly produced by the company TI located in USA. The DSP chip can be selected with DSP2812 or DSP28335. More details about the function of the two types of DSP can refer to the literatures [20-25].

The relay is connected in series with the FireWire. The DSP chip controls the relay on and off through the transistor drive circuit. On one hand, it is used to start acquisition of transient load fingerprints, and on the other hand it is used to respond to the on/off control 
commands of the upper platform to achieve elastic load on the demand side under the automatic response under management system.

The ZigBee wireless communication [26-27] module is connected to the DSP chip via a serial communication SCI interface to complete data communication with the intelligent interactive concentrator. The LCD liquid crystal serial port screen is used to display detection data and fingerprint recognition results.

The power supply unit includes an anti-surge protection circuit, an $\mathrm{AC} / \mathrm{DC}$ conversion module, a second-order filter circuit, a DC/DC conversion circuit, and finally outputs different voltage levels to maintain the normal power supply of each terminal unit.

\subsection{Hardware testing of the socket-type intelligent collection terminal}

Based on the hardware circuit diagram shown in Figure 4, we have developed a smart acquisition terminal prototype shown in Figure 5. We used this prototype to conduct experiments on the load fingerprints of typical household appliances. In the experimental process, we used electronic appliances (power lamps with a rated power of $12 \mathrm{~W}$ ) and resistance appliances (electric kettles with a rated power of $1500 \mathrm{~W}$ ) as an example. The measured steady-state operating current waveforms are shown in Figure 6.

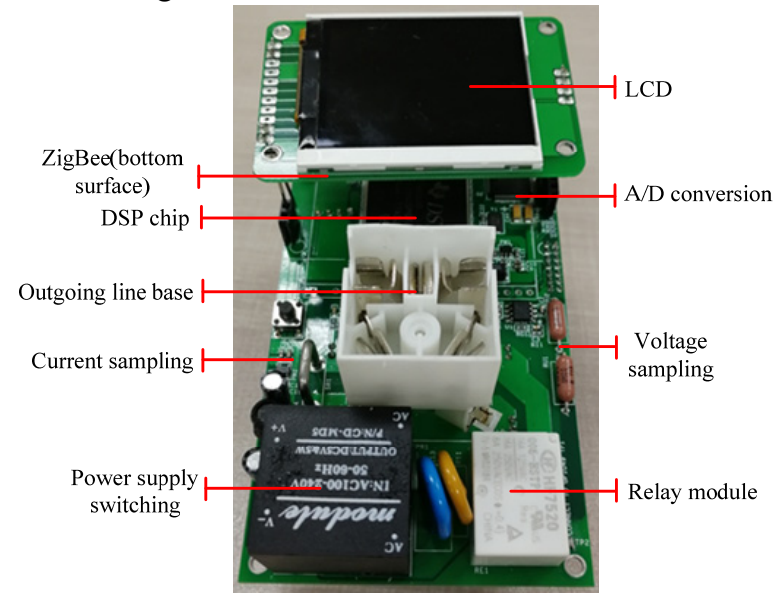

Fig. 5. Prototype of the intelligent collection terminal.
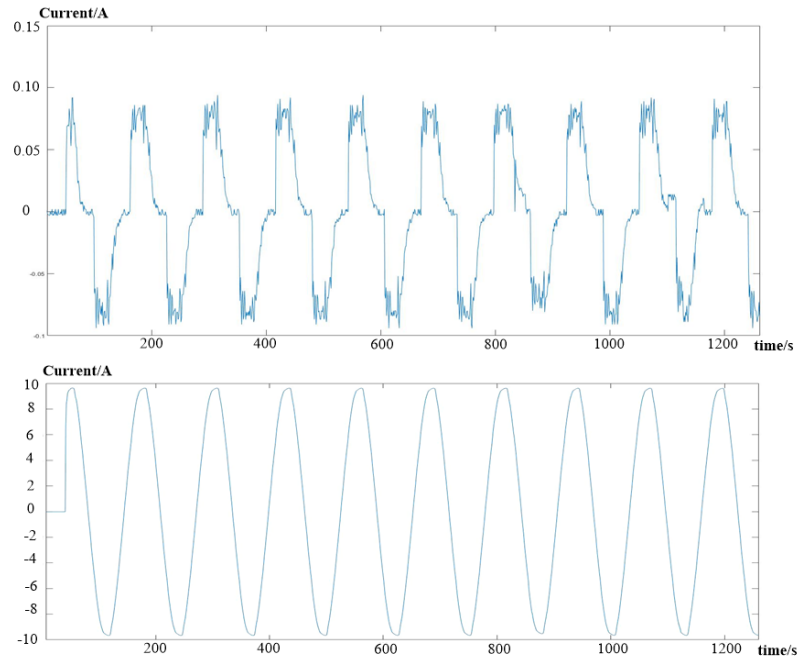

Fig. 6. Actual current waveforms of the fluorescent lamp and electric kettle.

In addition, we also conduct ZigBee wireless transmission performance tests on smart sampling terminal prototypes. We use the ZigBee wireless communication module based on the $\mathrm{CC} 2630$ radio frequency chip as the master node. The intelligent collection terminal prototype is used as the slave node. The master node is connected to the computer through the serial port conversion line. The baud rate of the serial port is set to $38400 \mathrm{bps}$. We use the computer's command transmission and data reception window to observe the data transmission results to test the wireless transmission performance of the prototype. After our tests, the experimental results obtained are shown in Table 1. In this table, 100k bytes are continuously transmitted for each experiment. Through this table, we found that the wireless transmission performance of the prototype developed satisfies the general requirements of ZigBee communication technology.

Table 1. Experimental results.

\begin{tabular}{cccc}
\hline $\begin{array}{c}\text { Numb } \\
\text { er }\end{array}$ & $\begin{array}{c}\text { Packet } \\
\text { length } \\
\text { /byte }\end{array}$ & $\begin{array}{c}\text { Interval between } \\
\text { packets } \\
/ \text { ms }\end{array}$ & $\begin{array}{c}\text { Bit error } \\
\text { ratio } / \%\end{array}$ \\
\hline 1 & 64 & 60 & 0 \\
2 & 64 & 50 & 0 \\
3 & 96 & 200 & 0 \\
4 & 96 & 100 & 0 \\
5 & 128 & 300 & 0 \\
6 & 128 & 200 & 0.013 \\
7 & 256 & 500 & 0 \\
8 & 256 & 400 & 0.046 \\
\hline
\end{tabular}

\subsection{Smart interactive concentrator development}

As a transit station for fingerprint data and control commands between the local smart collection terminal and the cloud management platform, the intelligent interactive concentrator shall support various communication technologies such as $\mathrm{ZigBee}, \mathrm{WiFi}$, and 
Ethernet to implement functions such as data upload and command response.

As illustrated in Figure 7, the schematic diagram of the unit structure of the intelligent interaction concentrator is similar to the installation of a typical non-intrusive detection device. The intelligent interaction concentrator is installed at the entrance bus and controls the access or bypass through the inlet and outlet air switches and the bypass air switch.

The functional units inside the device are basically similar to the smart acquisition terminals, but differ in the specific circuit design and device selection, mainly including: DSP core board, electrical measurement unit, high-power relay unit, clock and storage circuit, communication unit and power supply unit. Among them, the electrical measurement unit uses voltage transformers and current transformers to obtain sample values. The communication units include ZigBee modules, Wi-Fi modules, and Ethernet interfaces. The main circuit board is connected to an ARM-based tablet integrated machine via an RS232 serial data interface, and the integrated machine is loaded with load fingerprint management and application Android software to realize localized management based on load fingerprint recognition. Figure 8 shows the floor plan of the prototype that was actually developed.

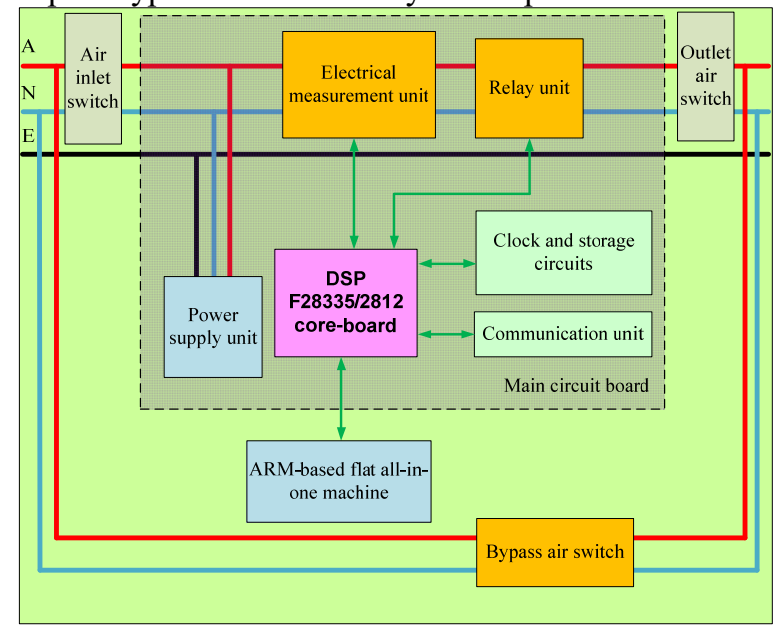

Fig. 7. Structure design of the intelligent interactive concentrator.

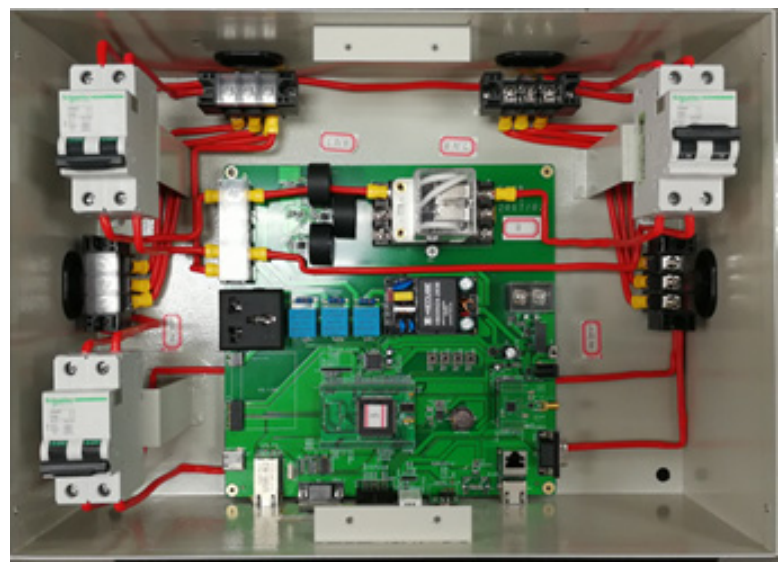

Fig. 8. Prototype of the intelligent interactive concentrator.

\section{Conclusion}

This paper first proposes the concept of load fingerprinting and its composition. Then, based on the information physics fusion system, a hierarchical technical framework for load fingerprint management is designed. This architecture includes a local and a cloud platform, which constitutes a two-layer fingerprint management and application platform. This platform is conducive to solving the problem that the current nonintrusive detection technology is only limited to a given device, so as to promote the identification of load characteristics based on fingerprint data. Finally, this paper develops two kinds of key equipment, including a socket-type intelligent collection terminal and an intelligent interactive concentrator supporting multiple communication technologies. The two prototypes can provide solid hardware support for the realization of the technical architecture proposed in this paper.

The engineering application and promotion of the load fingerprint management framework proposed in this paper will effectively promote the power consumption behaviour analysis and power optimization control functions in the automatic demand side management system. The next step in this paper will be focused on the research of load identification based on fingerprint data and the development of corresponding load fingerprint management software.

\section{Acknowledgement}

The work is partially supported by the Key Science and Technology Projects of China Southern Power Grid (Grant No. GZKJQQ00000419).

\section{References}

1. C.K. Shi, B. Zhang, W.X. Sheng, et al. A discussion on technical architecture for flexible intelligent interactive power utilization, Power System Technology, 2013, 37(10): 2868-2874.

2. Y. Li, B.B. Wang, F.X. Li, et al. Outlook and thinking of flexible and interactive utilization of intelligent power. Automation of Electric Power Systems, 2015, 39(17): 2-9.

3. L. Luo, S. Huang, M. Liu. Framework design of smart power utilization system. In proceedings of China International Conference on Electricity Distribution. IEEE, 2010:1-5.

4. L.F. Cheng, Z.Y. Zhang, H.R. Jiang, et al. Local energy management and optimization: A novel energy universal service bus system based on energy Internet technologies. Energies, 2018, 11(5), 1160.

5. P. Palensky, D. Dietrich. Demand side management: demand response, intelligent energy systems, and smart loads. IEEE Transactions on Industrial Informatics, 2011, 7(3): 381-388.

6. L.F. Cheng, T. Yu. Development of three-phase smart meter based on dual ATmega128L and its 
applications in power quality. Taylor \& Francis Group, 2014, 2: 49-54.

7. L.F. Cheng, L.F. Yin, B. Zhou, et al. Development of new multi-function power quality on-line monitoring device. Advanced Materials Research, 2014, 971-973: 928-933.

8. L.F. Cheng, T. Yu. A new kind of smart dual DSP2812 power quality monitor based on dedicated users. Advanced Materials Research, 2014, 971-973: 2004-2012.

9. S.S. Venkata, E. Sortomme. Using advanced measurement systems for microgrid protection. IEEE PES Innovative Smart Grid Technologies Conference. IEEE, 2012: 1-1.

10. Y.C. Ke. Research on the characteristic and response potential of household electric appliance load. Master Thesis, East China Jiaotong University, Nanchang, China, 2017.

11. Q. Q. Liu. Research and application of residents load signature. Master Thesis, Shanghai University of Electric Power, Shanghai, China, 2014.

12. X.Y. Yu, B. Liu, W.P. Luan. Nonintrusive residential load monitoring and decomposition technology. Southern Power System Technology, 2013, 7(04): 1-5.

13. L.F. Cheng, T. Yu, X.S. Zhang, et al. Cyberphysical-social systems based smart energy robotic dispatcher and its knowledge automation: framework, techniques and challenges. Proceedings of the CSEE, 2018, 38(1): 25-40.

14. S. Ma, Z. Xu, L.M. Wang. Set theory based modeling method of cyber physical system for power grid. Automation of Electric Power Systems, 2017, 41(6): 1-5.

15. R. Rajkumar, I. Lee, L. Sha, et al. Cyber-physical systems:the next computing revolution. In Proceedings of the Design Automation Conference. IEEE, 2010: 731-736.

16. J. Lee, B. Bagheri, H.A. Kao. A Cyber-Physical Systems architecture for Industry 4.0-based manufacturing systems. Manufacturing Letters, 2015, 3:18-23.

17. L. Sha, S. Gopalakrishnan, X. Liu, et al. Cyberphysical systems: a new frontier. In Proceedings of the IEEE International Conference on Sensor Networks, Ubiquitous, and Trustworthy Computing. IEEE Computer Society, 2008:1-9.

18. J. Shi, J. Wan, H. Yan, et al. A survey of cyberphysical systems. In Proceedings of the International Conference on Wireless Communications and Signal Processing. IEEE, 2011:1-6.

19. B. Liu. Non-intrusive power load monitoring and disaggregation technique. Master Thesis, Tianjing University, Tianjing, China, 2014.

20. L.F. Cheng, T. Yu. Grounding wire detection based on three-phase symmetrical voltage injection method for distribution network. Electric Power Automation Equipment, 2017, 37(3): 19-25.
21. W.L. Yang, T.B. Jang. Hardware design of digital AC servo system based on DSP2812. In Proceedings of the International Conference on Intelligent Computing and Integrated Systems. IEEE, 2010: 337-340.

22. L.F. Cheng, B. Zhou, T. Yu. Design and implementation of energy-saving potential automatically detecting online and rapid energy audit intelligent system. Power System Protection and Control, 2014, 42(14):105-111.

23. Y.S. Kung, P.G. Huang. High performance position controller for PMSM drives based on TMS320F2812 DSP. In Proceedings of the IEEE International Conference on Control Applications. IEEE, 2004, 1: 290-295.

24. L.F. Cheng, J.F. Peng, Y.X. Li, et al. Development of special users' energy saving potential intelligent diagnosis detector based on DSP. Electrical Measurement \& Instrumentation, 2014, 51(18): 9297.

25. P. Palacký, P. Hudeček, D. Slivka, et al. Online diagnostic and control unit of vehicle induction motor based on TMS320F28335 DSP. In Proceedings of the Power Electronics and Motion Control Conference. IEEE, 2010: T9-66-T9-71.

26. W. Wang, G. He, J. Wan. Research on Zigbee wireless communication technology. In Proceedings of the International Conference on Electrical and Control Engineering. IEEE, 2011:1245-1249.

27. Z. Qi, Y. Zhang, L. Zhao, et al. A new wireless communication system based on ZigBee technology. In Proceedings of the International Conference on Photonics and Image in Agriculture Engineering. International Society for Optics and Photonics, 2009: 96. 